\title{
Comprehending negation: A study with adults diagnosed with high functioning autism or Asperger's syndrome
}

\author{
REBECCA SCHINDELE, JANA LÜDTKE, and BARBARA KAUP
}

\section{Abstract}

We investigated whether readers with high-functioning autism or Asperger's syndrome $(H A / A S)$ differ from normal controls with respect to pragmatic aspects of negation processing. We presented short stories to two groups of readers, a group of individuals diagnosed with $H A / A S$ and a group of normal controls. The final sentence of each story either affirmed or negated a particular proposition, which in the pragmatically felicitous context corresponded to a highly plausible assumption for the situation at hand, but in the pragmatically infelicitous context to an implausible assumption. In line with our predictions, the group of healthy controls read the negative but not the affirmative target sentences more slowly in the pragmatically infelicitous than in the pragmatically felicitous contexts. In the pragmatically felicitous context, reading times for negative sentences were as fast as those for affirmative sentences. In contrast, for the clinical group, the context had no effect: Reading times for the negative target sentences were longer than those of the affirmative target sentences in both context versions. These results indicate that individuals with $H A / A S$ indeed differ from normal controls with respect to negation processing. Moreover, these results are in line with the more general hypothesis that the differences between normal individuals and those with $H A / A S$ concern pragmatic aspects of language processing.

\section{Introduction}

It is widely assumed that the contexts in which negative utterances occur are rather limited (e.g., Halliday \& Zoe 1993; but see Mehl and Pennebaker, 2003 for the specifics of its prevalence). Typically, negative statements are uttered when the negated proposition was either explicitly mentioned before by one of the discourse partners or at least constitutes a 
plausible assumption in the respective context (Clark \& Clark 1977; Colston 1999; Heinemann 1983; Schmidt 1973). Thus, negation is used to communicate deviations from what was previously assumed or expected (e.g., Givón 1978; for a different view, see Giora 2006). For the listener, a negative sentence "not $p$ " therefore not only communicates that $p$ is considered false by the speaker but also that the speaker thinks that the listener may believe that $\mathrm{p}$. Accordingly, when uttered in a context in which the listener indeed believes $\mathrm{p}$, or at least can understand why the speaker may think so, the negation is pragmatically felicitous and should be relatively easy to process. In contrast, when uttered in a context in which the listener did not believe $\mathrm{p}$, and cannot find reasons why the speaker may think so, then negating $\mathrm{p}$ is pragmatically infelicitous and should lead to comprehension difficulties. More specifically, in the latter case, the listener must retroactively activate the assumption that the speaker apparently wishes to correct before he or she can do any kind of revision. In addition, the listener may be confused by the apparent mismatch between his or her own beliefs and the beliefs that the speaker ascribes to him or her. Take for instance the sentence The children were not wearing live vests when uttered in the context of a regular morning in a children's day-care centre. As there is no reason to expect the children to wear life vests, the negative sentence will probably lead to processing difficulties on the part of the listener, despite the fact that it is most probably true for the situation at hand (see Levine \& Hagaman 2008, this issue). The reason is that the listener didn't expect the children to wear life vests and cannot understand why the speaker may consider it possible that he/ she might do so. In contrast, the same sentence uttered in the context of an airplane's emergency landing, the sentence probably does not lead to processing difficulties. The hypothesis that the children were wearing life vests is plausible for the situation at hand, and the listener therefore should not be surprised that he or she is informed that this plausible assumption does not hold for the particular situation at hand.

In line with these considerations past studies investigated the processing of negation inside or outside of a pragmatically felicitous context. In 1965, Wason tested the context-dependency of negation processing with respect to non-linguistic contexts. He presented participants pictures of eight circles, seven in one color and one in another (e.g., circle 3). They were then asked to complete affirmative or negative sentence fragments. Negative fragments (e.g., Circle No 3 is not ...) took longer to complete than affirmative fragments (e.g., Circle No 3 is ...), but this difference was significantly smaller when the negative sentence referred to the circle with the exceptional color than when it referred to one of the seven other circles. Thus, participants seemed to profit from an adequate context 
when processing negative sentences (but see Arroyo 1982, for some qualifications.) There are also studies that tested the context dependency of negation processing with respect to linguistic contexts. For instance, Glenberg, Robertson, Jansen, \& Johnson-Glenberg (1999), showed that the processing of negative sentences can be facilitated when the prior context highlights the attribute dimension that is referred to in the negated proposition (e.g., color of the couch for The couch is not black). Similarly, Lüdtke and Kaup (2006) demonstrated that the processing of a negative sentence is significantly facilitated when the negated proposition was explicitly mentioned as a possibility in the prior context. No significant facilitation was observed for affirmative sentences, which suggests that facilitation was stronger in the negative than in the affirmative conditions and therefore allows ruling out a simple priming explanation of the results. In addition, Lüdtke and Kaup (2006) provided evidence that negation processing is also relatively easy when the linguistic context strongly suggests the negated proposition. Under these circumstances negative sentences require the same amount of processing time as their affirmative counterparts. In contrast, when the context only weakly suggests the negated proposition, negative sentences require significant more processing time than their affirmative counterparts. Taken together, these findings suggest that for normal readers negative sentences are relatively hard to process when presented in a pragmatically infelicitous context, i.e., in a context in which the negated proposition was neither particularly plausible nor previously uttered by one of the discourse partners. In contrast, when presented in a felicitous context, processing of negation is facilitated, and in some cases even as easy as the processing of the corresponding sentences without negation (e.g., Wason 1965; and Glenberg et al. 1999; Lüdtke \& Kaup 2006, respectively).

What does this implicate with respect to negation processing in individuals with autism? Autism is a well know developmental disorder with symptoms that start occurring in early childhood. One of the diagnostic criteria for an autism disorder in current practice is a chronic impairment in social relations (Baron-Cohen 1988; Fein, Pennigton, Markowitz, Braverman, \& Waterhouse 1986; Sodian 2005; Tager-Flusberg 1999). Deficits in the ability to participate in two-way reciprocal social interaction persist throughout the whole lifespan of autistic individuals, independent of the heaviness of their impairments along the autism spectrum (Baron-Cohen 1988). For speaking autistic children and adults, this deficit is observable in the communicative use of language. Despite considerate within-population variability, a characteristic pattern has begun to emerge in the studies concerned with the communicative use of language in individuals diagnosed with the autism disorder: Whereas for normal 
individuals, an essential motivation to communicate is a desire to share intentions, thoughts and emotions with others, people with autism rarely use language as a means of providing information to others that is new to them or for obtaining information from others that is new to oneself (Tager-Flusberg 1996). Although most reach adequate test results in language tests that measure syntactic or semantic aspects of language processing (Szatmari, Tuff, Finlayson, \& Bartolucci 1990; Tager-Flusberg 1985), individuals with high functioning autism and those diagnosed with Asperger's disorder (a mild form of the autism syndrome), usually violate conversational rules of acceptability and politeness when using language in social interaction (Baltaxe 1977; Tager-Flusberg 1981), and more generally show deficits in pragmatic aspects of language processing in production as well as in comprehension (e.g., Kelley, Paul, Fein, \& Naigles 2006; Loukusa 2007; Norbury \& Bishop 2002; Ozonoff \& Miller 1996; Ramberg, Ehlers, Nydén, Johansson, \& Gillberg 1996). In comprehension, individuals with high functioning autism or Asperger's syndrome (HA/AS) exhibit problems especially in cases in which coming up with an adequate interpretation requires taking into account the intentions of the speaker (Dennis, Lazenby, \& Lockyer 2001), reinterpreting the meaning of an utterance (Ozonoff \& Miller 1996; Rumsey \& Hanahan 1990) or integrating information from different sources (e.g., Jolliffe \& BaronCohen 1999a, 1999b, 2000; Noens \& van Berckelaer-Onnes 2005; Norbury \& Bishop 2002). Accordingly, individuals with HA/AS have problems with understanding indirect requests (e.g., Ozonoff \& Miller 1996), idioms (Kerbel \& Grunwel 1998), metaphors (Happé 1993, 1995), jokes (Emerich, Creaghead, Grether, Murray, \& Grasha 2003; Ozonoff \& Miller 1996), and irony (Happé 1993, 1995). They tend to interpret utterances overly literal (e.g., Kaland, Møller-Nielsen, Callesen, Mortensen, Gottlieb, \& Smith 2002) and show deficits in establishing a locally and globally coherent discourse representation (Jolliffe \& Baron-Cohen 2000).

Although researchers more or less agree that people with HA/AS have difficulties mainly with respect to pragmatic aspects of language, the underlying causes are still a matter of debate. According to one very commonly suggested explanation, people with HA/AS are characterized by a weak central coherence, with the result being that they attend to small bits of information rather than to large, globally coherent patterns of information (Frith 1989; Frith \& Happé 1994; Happé \& Frith 2006). In the non-verbal domain, weak central coherence can account for the superior performance of people with HA/AS in the embedded-figures test (Jolliffe \& Baron-Cohen 1997) and the block-design test (Shah \& Frith 1993). The pragmatic difficulties in the verbal domain can also be accounted for: 
People with HA/AS tend to process each piece of information in isolation without routinely taking into account contextual information, as for instance the particular communicative situation or the intentions of their discourse partners. As a result they interpret the utterances overly literal and without an adequate understanding of the underlying speech acts. Another commonly suggested explanation assumes that people with HA/AS suffer from a theory-of-mind deficit (Baron-Cohen, Leslie \& Frith 1985; Baron-Cohen, Tager-Flusberg \& Cohen 2000).

According to this account, people with HA/AS have difficulties in understanding other people's mental states, especially acknowledging the fact that the mental states of others may differ from their own mental states (Baron-Cohen 1988; Sodian 2005). In the non-verbal domain, this account can explain why individuals with HA/AS score relatively poorly compared with a control group of healthy adults when presented with tasks that require such an ability of mentalizing, like false-belief tests, even if the groups are matched with regard to age and general intelligence (e.g., Baron-Cohen 2000; Frith \& Happé 1994). In a false belief-test, participants need to attribute a false (and therefore different) belief to another person (e.g., the chocolate is in the cupboard) and use this to predict the person's behavior (e.g., she will look for the chocolate in the cupboard) (Wimmer \& Perner 1983). Normal children are typically able to solve this kind of task from age 4 on. In contrast, autistic children of normal intelligence often fail in this kind of test, indicating an impairment in distinguishing their own beliefs from someone else's beliefs (BaronCohen 1993; Baron-Cohen et al. 1985). In the verbal domain, the theory-of-mind-deficit hypothesis is obviously well in line with the observation that people with $\mathrm{HA} / \mathrm{AS}$ have special difficulties in situations in which coming up with an adequate interpretation of an utterance requires taking into account the communicative situation and the intentions of the speaker.

Let us now return to the question of what these considerations imply with respect to negation processing in people with HA/AS. As we mentioned in detail above, there is evidence that normal readers interpret a negative utterance as communicating not only information with respect to the state of affairs under consideration but also with respect to what the speaker thinks that the listener may consider a plausible alternative. As a result, negative utterances are especially difficult to process in a context in which the negated proposition does not correspond to a plausible alternative. If individuals with $\mathrm{HA} / \mathrm{AS}$ do not take into account contextual information when interpreting an utterance (weak-central-coherence account), or are unable to reason about the mental state of their discourse partner (theory-of-mind-deficit account), then these individuals 
can be expected to differ greatly from normal controls with respect to the processing of negation. More specifically, for people with HA/AS it should be relatively irrelevant whether a negative sentence is uttered in a context in which the negated proposition constitutes a plausible alternative (pragmatically felicitous context) or not (pragmatically infelicitous context).

To illustrate, for a healthy individual, an utterance such as "The door is not closed," while communicating that the door is open (Kaup, Lüdtke, Zwaan 2006), may further suggest that the speaker assumes that the listener believes or suspect that the door is closed (Clark \& Clark 1977; Givòn 1978). If this is what the listener believes, or if the listener at least understands why the speaker thinks that this is what he or she believes, then the sentence is relatively easy to process. If not, the sentence should be relatively hard to process, be it because the comprehender realizes that the negation is not used as a means for correction or because the comprehender is confused about the apparent mismatch between what he or she considers plausible and what the speaker assumes that he or she considers plausible. In contrast, for an individual with weak central coherence, the negative sentence is not related to the context in which it occurs and it should therefore not matter whether the negated proposition is plausible or implausible in the present context. Similarly, for an individual with a theory-of-mind deficit, an utterance such as "The door is not closed" only communicates that the door is open but does not imply any conclusions with respect to the mental state of the speaker. Thus, according to both explanations of the autism disorder, the negative version may or may not be harder to process than the corresponding affirmative "The door is open", but should not be particularly facilitated in case the individual expected the door to be closed.

Based on these assumptions, we hypothesized that processing times for negative sentences should not be influenced by whether or not the sentences are presented in a pragmatically felicitous context for individuals with HA/AS. In contrast, for healthy controls, processing times should be faster in pragmatically felicitous than in pragmatically infelicitous contexts, as reported in previous empirical studies (e.g., Glenberg et al. 1999; Lüdtke \& Kaup 2006; Wason 1965). To test these hypotheses, we constructed short stories describing everyday events involving two characters (e.g. two people getting ready for a walking tour). The descriptions were such that they strongly implied a particular inference (e.g., that the protagonists were putting on special boots for walking). The final sentence of each story either confirmed or disconfirmed this inference by means of an affirmative or negative syntactic structure, respectively (e.g., She said that she had / had not put on her hiking boots). In the negative version, 
the sentence denied a plausible assumption (i.e., the inference that was presumably drawn when participants read the previous sentences). The negation should therefore be pragmatically adequate, and accordingly, the sentence should not be particularly hard to process, at least not for healthy readers. Measuring this expected facilitation effect due to a pragmatically felicitous context, requires a control condition. We therefore constructed a second version of each story. In this version, a slightly different event was being described, and importantly, the description did not imply the proposition that was confirmed or denied in the target sentence of the story. For instance, instead of preparing for a walking tour, the protagonists in the second version of the story were getting ready to go out to a restaurant for dinner. As it is highly unlikely that they are putting on their hiking boots for this occasion, the negative sentence that denies this proposition should be pragmatically infelicitous. Accordingly, the negated target sentence should be relatively hard to process in this version, at least for normal readers who can be expected to take the pragmatics of negation into account. It should be noted that affirmative sentences are assumed to be much less contextdependent (Wason 1965). Thus, whether or not the proposition that is being confirmed in the affirmative target sentence was implied by the linguistic context should not matter as much for the affirmative versions of the stories.

Taken together, for normal readers we expect to find an interaction between the polarity of the target sentence and the context version of the story: Whereas the context should not have much effect on the processing of the affirmative target sentences, negative target sentences should be easier to process when the context implies the negated proposition than when the context does not imply this proposition. In contrast, for readers with HA/AS, who supposedly are insensitive to the pragmatics of negation, the processing of negative sentences should be relatively contextindependent as well. In predicting that HA/AS readers do not exhibit a context-dependency when processing the negative target sentences we deliberately leave open whether they will have general difficulties with negation or no difficulties at all. In principle it seems possible that readers with HA/AS have difficulties with negative sentences for reasons other than pragmatic adequateness. In this case, we expect to find a significant polarity effect of comparable magnitude in both context versions because readers with HA/AS should not profit from a pragmatically felicitous context. Alternatively, negative sentence may pose no special processing difficulties for individuals with HA/AS. In this case, we expect to find no polarity effect in neither of the context versions because HA/AS readers should not suffer from a pragmatically inadequate context. 


\section{Experiment}

\subsection{Method}

2.1.1. Participants. In the clinical group we combined the two diagnoses of high functioning autism and Asperger's disorder. The clinical group consisted of 19 participants. Fifteen of them were juvenile trainees of the Vocational Training Center at Oberlin House in Potsdam, Germany, diagnosed with Asperger's disorder or High-functioning autism. They were between 15 and 20 years old. The remaining four participants were adults between 25 and 53 years of age, all diagnosed with Asperger's disorder. All diagnoses were made by local mental health professionals prior to the experiment. All participants were native speakers of German, and participated in the experiment on a voluntary basis.

The control group consisted of 20 participants. Fifteen were students at the University of Technology, Berlin, between 20 and 30 years of age. The remaining five were pupils at several secondary schools. They were between 17 and 18 years old. All control participants were native speakers of German with no learning disability or other neurological disorder. They participated for a reimbursement of 4 euro.

2.1.2. Materials. The materials consisted of 36 short German stories, 24 of which were used as experimental items, and 12 as filler items. The experimental items were constructed according to the following schema (see Table 1): An introductory paragraph, which included between one

Table 1. Sample Text (Roughly translated from German to English)

\begin{tabular}{ll} 
Introduction & $\begin{array}{c}\text { Thomas and Conny are spending a weekend in the mountains in } \\
\text { order to relax from all the stress during the week. }\end{array}$ \\
\hline $\begin{array}{l}\text { Variation sentence } \\
\text { Relevant Inference }\end{array}$ & $\begin{array}{r}\text { Today they decided to go on a walking tour for which they are } \\
\text { currently getting dressed. } \\
\text { This evening they decided to go to a nice restaurant for which they } \\
\text { are currently getting dressed. }\end{array}$
\end{tabular}

Filler sentence Thomas wants to leave and is starting to get impatient. Finally, Conny is ready to go.

\section{Target Sentence \\ affirmative negative}

Question
She tells Thomas that she put on her hiking boots.

She tells Thomas that she didn't put on her hiking boots.

Are Thomas and Conny on a weekend trip? (Yes) 
and three sentences, specified the setting of the story and introduced the main characters. The subsequent sentence described a particular event that the main characters were involved in (e.g., getting dressed). This "variation sentence" was available in two versions that differed only minimally with respect to the wording, but implied different inferences. For instance, if someone is getting dressed to go walking, she is likely to put on hiking boots (inference A). In contrast, if she is getting dressed to go to a restaurant, possibilities are high that she is putting on a pair of finer shoes (inference B). Up to three filler sentences followed the variation sentence. These filler sentences elaborated on the event described in the variation sentence and were the same in all versions. The subsequent "target sentence" either affirmed or negated inference A. Thus, in version "inference A" the negative target sentence denied a plausible proposition, whereas in version "inference B" it denied an implausible proposition. Accordingly, version "inference A" should constitute a pragmatically felicitous context for the negative target sentence, but version "inference B" should constitute a pragmatically infelicitous context. For reasons of readability, we will refer to the two context versions with the terms "relevant-inference" condition and "other-inference" condition in what follows.

Twelve filler stories of comparable lengths and topics as the experimental stories were constructed and served to obscure the manipulation. Six of the filler stories ended with a negative sentence, and the remaining six ended with an affirmative sentence. For each of the filler stories, and for eight of the twenty-four experimental stories, simple comprehension questions were constructed. Half of these required a "yes"-response, and the other half a "no"-response.

2.1.3. Design and procedure. The design and the procedure of the experiment was the same for the two groups of participants (clinical and control). All participants read all 24 experimental items intermixed with all 12 filler items. The 24 experimental items were assigned to four lists that counterbalanced items and conditions. Each list contained 6 stories in each of the four versions, and each participant saw one of these four lists. Thus, we employed a 2 (group: clinical vs. control) $\times 2$ (polarity of target sentence: affirmative vs. negative) $\times 2$ (context: relevant inference vs. other inference) $\times 4$ (list) design with repeated measurement on the variables "polarity" and "context".

Each participant saw 12 stories in the "relevant inference" version and 12 stories in the "other inference" version. Half of each of these ended with an affirmative target sentence and the remaining half ended with a negative target sentence. In addition, each participant saw 6 filler stories 
ending with a negative sentence and 6 filler stories ending with an affirmative one. The order in which these stories were presented was random for each participant.

Participants were tested individually. At the beginning of the experimental session, the participants were presented with a written instruction regarding the experimental procedure, and then practiced the procedure in two practice trials. The stories were presented sentence-by-sentence, self-paced by the participants pressing the space bar. Pressing the spacebar after reading the final sentence of a story elicited in 20 out of 36 trials the presentation of the comprehension question which was written in a different color than the stories proper. Participants responded to the comprehension question by pressing the "."- or " $x$ "-key (marked with " $y$ " and " $n$ ", respectively). They received feedback on their answers. The experimental session lasted approximately 20 minutes.

\subsection{Results and discussion}

In the clinical group, the data of two participants were discarded because they made mistakes on half of the experimental comprehension questions, and their mean reading times deviated more than 1.5 standard deviations from those of the remaining participants. Mean percentages of correct responses to the comprehension questions in experimental items and mean reading times of the target sentences were submitted to 2 (group) $\times$ 2 (polarity of target sentence) $\times 2$ (context) $\times 4$ (list) analyses of variance (ANOVAs) with repeated measurement on the factors "polarity" and "context", in both the by-participant analysis $\left(F_{1}\right)$ and the by-items analysis $\left(F_{2}\right)$.

2.2.1. Percentage correct. The mean percentages with which subjects correctly responded to the comprehension questions of experimental items in the different conditions are displayed in Table 2. The statistical analyses revealed no main effects of context or polarity (all $F s<1$ ), nor an interaction of polarity and context (all $F s<1$ ). Most important, there

Table 2. Comprehension-Question Accuracy for Experimental Items (Mean Percentage Correct)

\begin{tabular}{llllll}
\hline & \multicolumn{2}{c}{ Relevant inference condition } & & \multicolumn{2}{c}{ Other inference condition } \\
\cline { 2 - 3 } \cline { 6 - 7 } & affirmative & negative & & affirmative & negative \\
\hline Clinical group & $82 \%$ & $88 \%$ & & $88 \%$ & $88 \%$ \\
Control group & $85 \%$ & $90 \%$ & & $90 \%$ & $85 \%$ \\
\hline
\end{tabular}


was no main effect of group $(F 1, F 2<1)$, nor any interactions between group and the variables polarity, context, or context-by-polarity (all $F s<1)$. This indicates that the two groups did not differ with respect to an overall, relatively coarse measure of comprehension accuracy. In line with this interpretation, the two groups also did not differ in accuracy with respect to the comprehension questions of the filler stories in the experiment: Participants in the control group answered $89 \%$ correct and participants in the clinical group $90 \%(t 1(35)=0.3, p>.50 ; t 2(11)=$ $0.5, p>.50)$.

2.2.2. Reading times. Reading times longer than $20000 \mathrm{~ms}$ were omitted $(\mathrm{N}=4)$. In determining outliers within the remaining reading times, we took differences among the participants and the four versions into account. First, the reading times of each participant in each condition were converted to $\mathrm{z}$ scores. Then reading times with a $\mathrm{z}$ score that deviated more than $\times$ standard deviations from the mean $\mathrm{z}$ score of the respective participant in the respective condition were discarded, with $\times$ depending on the number of observations per condition, as suggested by Van Selst and Jolicoeur (1994). As in our design there were six observations per participant and condition, $\times$ was set to 1.84 . For the three cells with only five observations (i.e., the cells where one response time was above $20000 \mathrm{~ms}$ and therefore deleted) $\times$ was set to 1.68 . This procedure eliminated less than $2.2 \%$ of the data in the clinical group and $4.6 \%$ of the data in the control group. The means of the remaining reading times in the four conditions are displayed in Figure 1.

The observed reading time pattern is quite clear. For the clinical group, negative target sentences were read more slowly than affirmative target sentences in both context versions. In contrast, for the control group, negative target sentences were read more slowly than affirmative target sentences mainly in the other-inference condition. These differences between the two groups were not reflected in the overall ANOVA in which group was a between-participant factor. However, the differences were reflected very nicely in the separate analyses conducted for the two groups. The overall ANOVA produced a highly significant main effect of group, with participants in the control group exhibiting faster reading times than the participants in the clinical group $\left(F_{1}(1,29)=33.7, p<.001 ; F_{2}(1,20)=\right.$ $225.1, p<.001$ ), and a highly significant main effect of polarity, with faster reading times in the affirmative than in the negative conditions $\left(F_{1}(1,29)=9.0, p<.01 ; F_{2}(1,20)=16.5, p=.001\right)$. There was no main effect of context $\left(F_{1}(1,29)=1.0, p=.32 ; F_{2}(1,20)=1.2, p=.28\right)$, nor a context-by-polarity interaction $\left(F_{1}(1,29)=1.2, p=.28 ; F_{2}(1,20)=\right.$ $1.5, p=.23)$. The group-by-context-by-polarity interaction was also not 

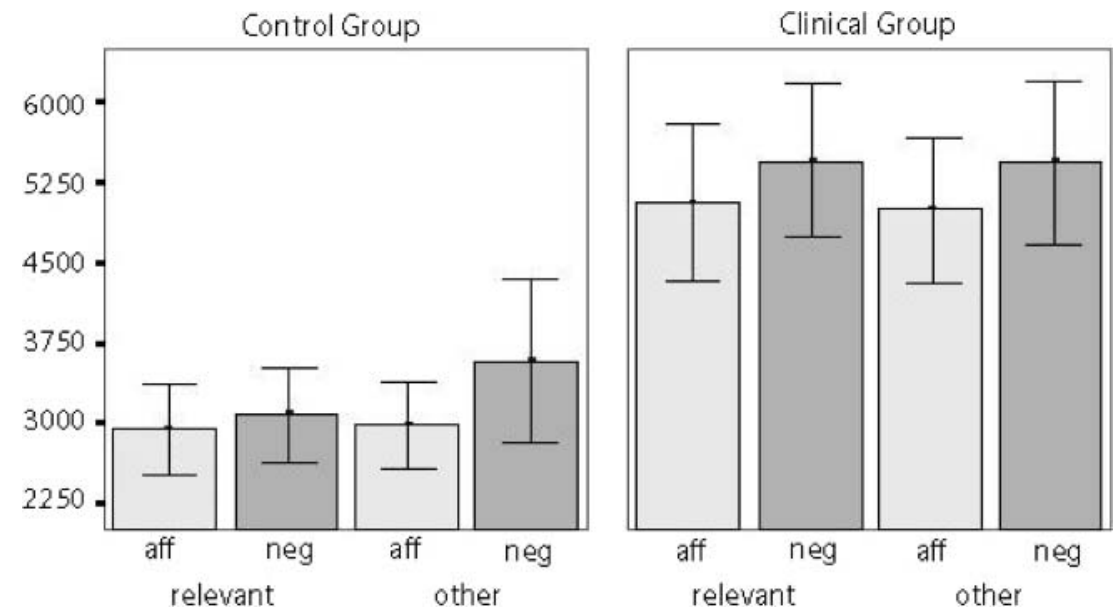

Figure 1. Mean reading times of the target sentence as a function of sentence polarity and context version. Error Bars represent the confidence interval of $95 \%$

significant $\left(F_{1}<1 ; F_{2}(1,20)=1.6, p=.23\right)$. According to the hypotheses, we nevertheless conducted separate analyses for the two groups. For the clinical group, there was a significant main effect of polarity $\left(F_{1}(1,13)=\right.$ $\left.4.7, p<.05, F_{2}(1,20)=8.4, p<.01\right)$, but neither a main effect of context, nor a context-by-polarity interaction (all $F \mathrm{~s}<1$ ). For the control group, there was a main effect of polarity which was marginally significant in the by-participants analysis and significant in the by-items analysis $\left(F_{1}(1,16)=4.2, p=.06, F_{2}(1,20)=5.6, p<.05\right)$. In contrast to the clinical group, there was also a polarity-by-context interaction, which was significant only in the by-items analysis, $\left(F_{1}(1,16)=2.4, p=.14\right.$, $\left.F_{2}(1,20)=5.1, p<.05\right)$. The main effect of context was not significant $\left(F_{1}(1,16)=1.9, p=.18, F_{2}(1,20)=1.7, p=.21\right)$. Planned comparisons conducted for the negative and affirmative target sentences in the control group confirmed the predictions: Whereas the reading times for affirmative sentences were not affected by the context (both $t<|1|$ ), negative target sentences were read significantly more slowly in context version "other inference" than they were in context version "relevant inference" $\left(t_{1}(1,19)=1.8, p<.05\right.$, one-tailed; $t_{2}(1,23)=1.7, p<.05$, one-tailed). This result fits nicely with the hypothesis that participants in the control group profited from an adequate context when processing the negative target sentences. The relevant-inference version, implying the negated proposition, constitutes a felicitous context for a negative sentence, and accordingly processing the negated target sentences in this version is relatively easy. In contrast, the other-inference version, not implying the 
respective proposition, constitutes an infelicitous context, and accordingly processing the negated target sentences in this version is relatively hard. The fact that no context effect obtained for the group of readers with $\mathrm{HA} / \mathrm{AS}$ is in line with the hypothesis that individuals with this disorder differ from normal controls with regard to pragmatic aspects of negation processing.

One could argue that the two groups did not only differ with respect to the diagnosis of HA/As but also with respect to age and general intellectual ability. In principle it seems possible that the different reading time patterns observed in the two group do not reflect differences between healthy readers and readers with $\mathrm{HA} / \mathrm{AS}$ but rather differences between younger and older readers or between readers with higher and lower intellectual ability. To obtain more information with respect to this alternative explanation, we conducted two post-hoc analyses. First, we repeated our analyses with only those participants who were between 20 and 30 years of age ( $\mathrm{N}=12$ for the control group; $\mathrm{N}=6$ for the clinical group). Second we repeated the analyses with only those participants who were enrolled as university students or already held a university degree $(\mathrm{N}=17$ for the control group; $\mathrm{N}=4$ for the clinical group). Thus, for these posthoc analyses, the two groups were similar as far as age and general intellectual ability was concerned. However, it should be noted that we consider these post-hoc analyses explorative at best because of the unequal number of subjects in the two groups.

The mean reading times for the four different conditions for the two age groups are displayed in Table 3. As can be seen, qualitatively the response time pattern looks very similar to the pattern observed for the whole group. The readers in the control group but not those in the clinical group have prolonged reading times for negative target sentences specifically

Table 3. Mean Reading Times for two soup groups of the two groups as a function of Context and Polarity (standard error of the mean in parentheses)

\begin{tabular}{llllll}
\hline & \multicolumn{3}{c}{ Relevant inference } & & \multicolumn{2}{c}{ Other inference } \\
\cline { 2 - 3 } & Affirmative & Negative & & Affirmative & Negative \\
& M (SE) & M (SE) & & M (SE) & M (SE) \\
\hline Control group & Homogenous with respect to Age & & \\
Clinical group & $5368(283)$ & $3180(294)$ & & $3016(272)$ & $3917(517)$ \\
\hline & & $5309(627)$ & $5493(823)$ & $5327(778)$ & $5453(997)$ \\
Control group & Homogenous with respect to Intellectual Ability & \\
Clinical group & 2964 (230) & $3108(237)$ & $3034(209)$ & $3744(406)$ \\
\hline
\end{tabular}


in the pragmatically infelicitous context. These observations were more or less also reflected in the statistical analyses. An overall ANOVA produced a highly significant main effect of group $\left(F_{1}(1,11)=151.4, p<\right.$ $\left..001 ; F_{2}(1,19)=87.3, p<.001\right)$. There was a trend towards a main effect of polarity $\left(F_{1}(1,11)=2.0, p>.15 ; F_{2}(1,19)=4.2, p=.05\right)$. The main effect of context, the context-by-polarity interaction, and the three-way interaction of group, context, and polarity were not significant (context $F_{1}(1,11)<1 ; F_{2}<1$; polarity: $F_{1}(1,11)=2.0, p>.15 ; F_{2}(1,19)=4.2$, $p=.05 ;$ context-by-polarity: $F_{1}(1,11)=1.3, p>.25 ; F_{2}(1,19)=3.0$, $p>.10$; group-by-context-by-polarity: $\left.F_{1}(1,11)<1 ; F_{2}<1\right)$. Separate analyses for the control group showed a significant main effect of polarity and a context-by-polarity interaction in the analyses by items (polarity: $F_{1}(1,9)=4.9, \quad p=.06 ; \quad F_{2}(1,20)=8.1, \quad p=.01 ; \quad$ context-by-polarity $\left.F_{1}(1,9)=2.0, p>.15 ; F_{2}(1,20)=4.4, p<.05\right)$. The main effect of context was not significant $\left(F_{1}(1,9)=2.7, p>.10 ; F_{2}(1,20)=2.6, p>.10\right)$. The separate analyses for the clinical group did not produce any significant effects (all $F s<1$ ). Planned comparisons conducted for the control group confirmed that polarity only affected the reading times in the pragmatically infelicitous context, i.e., the other-inference version (relevant inference: both $|t| \mathrm{s}<1$; other inference: $t_{1}(12)=2.5, p<.05 ; t_{2}(23)=3.2$, $p<.01)$, and context only affected reading times for negative target sentences (affirmative: both $|t| \mathrm{s}<1$ ); negative: $t_{1}(16)=2.0, p<.05$, onetailed; $t_{2}(23)=1.8, p<.05$, one-tailed).

Let us now turn to the analyses with a sample that is more homogeneous with respect to general intellectual ability. As mentioned above, in these analyses we included all those participants who were enrolled as university students or had completed a university degree.

The mean reading times in the four different conditions for the two groups are displayed in Table 3. As can be seen, the participants in the control group still show prolonged reading times specifically in the pragmatically infelicitous context. In contrast, in the clinical group, reading times for negative sentences in the pragmatically infelicitous context are not specifically prolonged. Although we are only looking at those participants who are comparable in general intellectual ability to the participants in the control group, the observed reading time pattern mimics the pattern that was observed for the whole group of readers with HA/AS, except that the increase in reading time in the negative contexts is much smaller. Thus, the observed response time pattern does not provide any indication that HA/AS readers with high intellectual ability differ from those with low intellectual ability with respect to a context-dependency of negation processing. Analyses of variance conducted with these subgroup data even showed the predicted three way interaction of group, 
context and polarity $\left(F_{1}(1,14)=4.9, p<.05\right)$. There were no main effects of context or polarity, and no context-by-polarity interaction (context: $F_{1}(1,14)=1.5, p>.20$; polarity: $F_{1}<1$; context-by-polarity: $\left.F_{1}<1\right)$, but of course a highly significant main effect of group $\left(F_{1}(1,14)\right.$ $=191, p<.01)$. In a separate analysis for the control group, a polarityby-context interaction was observed $\left(F_{1}(1,13)=4.6, p=.05 ; F_{2}(1,20)=\right.$ $6.4, p<.05)$. The main effect of polarity was significant in the analysis by items $\left(F_{1}(1,13)=2.9, p>.10 ; F_{2}(1,20)=5.2, p<.05\right)$ but the main effect of context did not reach significance in either of the analyses $\left(F_{1}(1,13)=3.6, p=.08 ; F_{2}(1,20)=2.1, p>.15\right)$. The separate analysis for the clinical group did not reveal any significant effects (all $F_{S}<1$ ). Planned comparisons conducted for the control group confirmed that polarity only affected the reading times in the pragmatically infelicitous context, i.e. the other-inference version (relevant inference: both $|t| \mathrm{s}<1$; other inference: $\left.t_{1}(16)=2.5, p<.05 ; t_{2}(23)=3.0, p<.05\right)$, and context only affected reading times for negative target sentences (affirmative: $\left|t_{1}\right|<1 ; t_{2}(23)=1.1, p>.25$; negative: $\left.t_{1}(16)=2.3, p<.05\right)$.

Thus, taken together, for the subgroup of readers with higher general intellectual ability, the results correspond exactly to our predictions: For healthy readers, but not for readers with HA/AS, negation processing is facilitated in pragmatically felicitous contexts. Yet, because of the small number of subjects in the clinical group, we do not want to overemphasize this result. At the very least however, these results seem promising for future studies in which high-intellectual-ability individuals with and without a diagnosis of HA/AS are compared with regard to negation processing.

\section{General discussion}

In the present paper we were concerned with the question of whether individuals with HA/AS differ from normal readers with respect to the processing of negative sentences. In particular, we were interested in whether individuals with HA/AS are sensitive to pragmatic aspects of negation. We investigated whether the context effect of negation processing that was observed in previous studies with normal readers would also be observed with readers with HA/AS. We presented short stories to two groups of readers, a group of individuals diagnosed with HA/AS, and a group of normal controls. The final sentence of each story either affirmed or negated a particular proposition, which in the pragmatically felicitous context corresponded to a highly plausible assumption for the situation at hand, but in the pragmatically infelicitous context to an implausible 
assumption. We measured the reading times of the affirmative and negative target sentences. In line with our predictions, the group of healthy controls read the negative but not the affirmative target sentences more slowly in the pragmatically infelicitous than in the pragmatically felicitous contexts. In the pragmatically felicitous contexts, reading times for negative sentences were as fast as those for affirmative sentences. In contrast, for the group of readers with HA/AS, the context had no effect: Reading times for the negative target sentences were longer than those of the affirmative target sentences in both context versions. These results suggest that individuals with HA/AS indeed differ from normal controls with respect to negation processing. In particular, these results are in line with the more general hypothesis that the differences between normal individuals and those with HA/AS concern pragmatic aspects of negation processing: Normal controls consider negation as a means of correcting false assumptions. Thus, when processing a negative sentence, they relate the sentence to the context in which it occurs, and as a result are sensitive to whether or not the proposition that is being negated corresponds to a plausible assumption for the situation at hand. If the negated proposition corresponds to a plausible assumption, processing is relatively easy. If it does not correspond to a plausible assumption, processing is difficult. The reason may be that comprehenders realize that the negation is not used in its typical function, namely as a means for correction. Alternatively, comprehenders may be confused about the apparent mismatch between what they themselves consider plausible and what the speaker assumes that they consider plausible. In contrast, for individuals with HA/AS, it apparently does not make a difference whether or not the negated proposition corresponds to a plausible assumption for the situation at hand.

There are several different possibilities for why this may be the case. An explanation that fits particularly well with the weak-central-coherence account of the HA/AS disorder (Frith 1989; Frith \& Happè 1994; Happé \& Frith 2006) is that people with this disorder do not routinely relate the negative sentence to the context in which it occurs, or at least show deficits in deciding which contextual factors are relevant and which are not, and as a result are not as sensitive to whether or not the negated proposition corresponds to a plausible assumption for the situation at hand as normal readers are. An alternative explanation is suggested by the theory-of-mind-deficit account (Baron-Cohen et al. 1985; 2000). According to this explanation, individuals with HA/AS do not draw any conclusions from the negated utterance with respect to the speaker's intention or mental state. As a result, processing of the negated sentence is not facilitated in case of a match between their own beliefs and those of the speaker. 
Several other explanations are conceivable also, even though they do not map as well onto the extant theoretical accounts of the autism disorder. First, individuals with HA/AS may simply not consider negation as a means for correction. If so, a negative sentence such as The door is not closed does not carry any additional information compared to the corresponding affirmative sentence with the same truth value (i.e., The door is open). In this case there is no good reason to expect any comprehension difficulties in cases in which the negation is not used as a means for correction. It should be noted, however, that this explanation would still be well in line with the more general hypothesis that individuals with HA/ AS differ from normal readers with respect to pragmatic aspects of negation processing. The same does not hold for the second additional explanation: Possibly, individuals with HA/AS differ from normal controls in that they do not enrich their discourse representation by elaborative inferences, or at least not to the same extent. If so, then the two contexts would not differ with respect to whether or not the negated proposition was part of the discourse representation that was available at the point in time at which the negative sentence was being processed. It might be argued that in this case there is no good reason to expect that processing of the negative sentence is more difficult in one or the other context. However, we do not consider this a valid alternative explanation for several reasons: First, there is evidence in the literature that individuals with HA/AS do draw elaborative inferences during comprehension (e.g., Ozonoff \& Miller 1996). But more importantly, even with the normal controls, we do not claim that the observed context effect necessarily reflects that comprehenders in all cases drew the respective inference when processing the context. In cases in which they did not spontaneously draw the inference, the negation in the target sentence probably retro-actively triggered a process for determining whether the negated proposition corresponds to a plausible assumption in the present context. In the pragmatically felicitous context, the negated state of affairs is likely according to world knowledge, and accordingly, comprehenders are able to understand why the speaker has used a negation to convey the respective information. No further processing difficulties should arise in this case. In contrast, in the pragmatically infelicitous context, the negated state of affairs is not likely according to world knowledge, and accordingly, comprehenders can be expected to continue being confused by the use of the negation in the target sentence. Along these lines of reasoning, we would expect that individuals with HA/AS also exhibit the context effect, if the only difference between them and the normal controls concerns the number of elaborative inferences that they are drawing during comprehension. The fact that no context effect was observed clearly speaks against the 
idea that the amount of inferencing is the decisive variable for whether or not a context effect is being observed.

As we implicitly mentioned above, there are two slightly different reasons for why negative sentences presented in pragmatically infelicitous contexts may lead to processing difficulties in normal readers. One possibility is that comprehenders notice that they themselves do not consider the negated proposition a plausible assumption in the present context, and accordingly realize that the negation is not used in its typical function, namely as a means for correction. This deviation from what is expected may lead to prolonged processing times. Alternatively, increases in processing time may reflect the fact that readers, when processing a negation, draw conclusions about the mental state of the speaker, namely that the speaker believes that the hearer may consider the respective proposition to be plausible. In case the comprehenders themselves do not consider the assumption to be plausible, and cannot find a reason why the speaker may think they do, they are confused about the apparent mismatch between their mental state and that of the speaker. This confusion alone may lead to increases in processing time. To our knowledge, there is not yet empirical evidence that allows a definite conclusion as to which of the two explanations is correct (but see Bonnefon \& Villejoubert 2007, for evidence from a reasoning study that points toward the latter hypothesis). Interestingly, the two theoretical accounts mentioned above differ with respect to the conclusions that can be drawn from the results of the present study with respect to this issue. According to the weak-centralcoherence account, readers with HA/AS do not show a context effect because they do not relate the negative sentence to the context in which it occurs. Thus, this account is neutral with respect to the exact nature of the reading time increases in infelicitous contexts in normal readers. In contrast, according to the theory-of-mind-deficit account, individuals with HA/AS differ from normal readers mainly in that they do not take into account the intentions of the speaker. Thus, the fact that normal readers $d o$ show a context effect when processing negative sentences, but individuals with HA/AS do not, reflects the fact that normal readers $d o$ take into account the mental state of the speaker, whereas individuals with HA/AS do not. Accordingly, the theory-ofmind-deficit explanation of the present results is more in line with the hypothesis that the prolonged reading times in the infelicitous contexts reflect the fact that readers notice a mismatch between what they themselves consider plausible and what the speaker apparently thinks that they consider plausible. Future research is necessary to decide why exactly the processing times for negative sentences are context-dependent for normal readers, as well as which of the theoretical accounts best 
accounts for the observed differences between normal readers and readers with HA/AS.

The experiment reported in this article focused on differences between normal readers and readers with HA/AS with respect to context effects in negation processing. We assumed that normal readers would have greater difficulties with negation in contexts in which the negated proposition does not constitute a plausible assumption. In contrast, for readers with HA/AS we expected that the context would not affect the difficulty of negation processing. This hypothesis was indeed borne out by the data. We deliberately left open whether readers with HA/AS would process negative sentences as easily as affirmative sentences, or whether they would show general difficulties with negation compared to affirmation. The reason is that there are several possibilities why negative sentences may be more difficult to process than affirmative sentences that go beyond the pragmatic aspects of negation. Thus, even if pragmatic violations are not an issue, negative sentences may still prose processing difficulties, be it because they are longer than affirmative sentences or syntactically more complex, or because they are associated with an unpleasant connotation (for an overview, see Wason 1972; Kaup, Zwaan, \& Lüdtke 2007). The fact that normal readers did not show a polarity effect in the pragmatically felicitous context may suggest that these factors do not play a significant role in negation processing for normal adults. In contrast, readers with HA/AS did show a polarity effect in both context conditions, and this indicates that these non-pragmatic factors may play a stronger role in negation processing for readers with HA/AS.

In this paper we were concerned with negation processing from the perspective of language comprehension. What can be hypothesized about the production of negation in normal vs. autistic individuals? For normal individuals, negation should be frequently used in contexts in which a particular highly plausible assumption turns out to be false for the situation at hand. After all, in a context in which a particular plausible assumption needs to be corrected, negation should be considered the adequate linguistic means for achieving this goal. In contrast, for individuals with HA/AS who presumably are insensitive to the pragmatic aspects of negation one may expect that negation is generally only rarely used, or that it is used but not as a linguistic means for correction. There are several other functions that negative sentences can fulfill in communication. For instance, negation is useful in situations in which not enough information is available for using an affirmative expression (cf. Beltran, Orenes, \& Santamaria, this issue) or as a means of mitigation, i.e., when a weakened assertion is to be made (cf. Fraenkel \& Schul this issue, Giora, Balaban, Fein, \& Alkabetz 2005; and Giora 2006, for a review). Furthermore, negation 
may be used for reasons of politeness (Giora et al. 2005) or for conveying understatement and irony (Giora, Fein, Ganzi, Levi, \& Sabah 2005). Obviously, of these functions, only the first two can be expected to be observed in autistic individuals, as the latter two certainly require theoryof-mind processes. As of yet there is not much empirical evidence on the production of negation, in particular not with respect to individuals with an autism disorder. The only relevant empirical evidence with respect to the production of negation in normal vs. autistic individuals that we are aware of comes from the developmental literature: Autistic children seem to follow normal children's pathway in the development of different meanings of negation in language production, but are significantly delayed. Normal children use negation for the disappearance, loss, or nonexistence of a referent very early on, then acquire negation as a means for rejecting a request, and only after that begin to use negation for denying the nature of a referent's property (e.g. "the horse hasn't got a saddle"), or a previously uttered assertion (e.g., "no, it is green." after somebody has stated that the object is blue) (cf. Bloom 1970; see also Watson 1979). In contrast, autistic children rarely use negation as a means for denial, even at later stages in the developmental process (Tager-Flusberg 1999; see also Shapiro \& Kapit 1978). These results suggest that children with autism are delayed in acquiring the denial function of negation, if they fully acquire it at all. This interpretation is in line with our hypotheses: If autistic individuals never fully acquire the denial function of negation, then it is of no surprise that they differ from normal controls with respect to the pragmatic aspects of negation. The question arises why the denial function of negation is not being fully acquired. One reason may be a theory-of-mind deficit. To deny the truth of another person's statement demands the idea that the other person may hold different beliefs. Future studies are necessary to obtain more information on the production of negation in normal and autistic individuals.

\section{Conclusion}

Readers with HA/AS differ from normal readers with regard to negation processing. Whereas normal readers are sensitive to whether or not the negated proposition corresponds to a plausible assumption for the situation at hand, readers with HA/AS are not. More specifically, for normal readers, processing times are prolonged for negative sentences relative to affirmative sentences only if the proposition that is being negated corresponds to an implausible assumption for the situation at hand. In contrast, for readers with HA/AS processing times are prolonged for negative 
compared to affirmative sentences regardless of context. Thus, readers with HA/AS differ from normal controls in that they do not profit from a pragmatically felicitous context when processing negative sentences. Whether the differences between the two groups of readers reflect the fact that readers with HA/AS do not routinely relate the negative sentence to the context in which it occurs (as assumed by the weak-centralcoherence account), or that individuals with HA/AS do not reason about the mental state of the speaker (as assumed by the theory-of-mind-deficit account) cannot be determined on the basis of the present data. However, what the results do indicate is that readers with HA/AS differ from normal readers mainly with respect to pragmatic aspects of language processing: Whereas normal controls are sensitive to the pragmatics of negation, readers with HA/AS are not. Negative sentences are difficult to process for readers with HA/AS even if they are presented in a pragmatically felicitous context.

\section{Acknowledgment}

Rebecca Schindele, Jana Lüdtke, and Barbara Kaup, Department of Psychology, University of Technology Berlin, Germany. Correspondence concerning this article should be addressed to Barbara Kaup (barbara. kaup@tu-berlin.de). This work was supported by grant KA1389/3-1 to B. Kaup. We would like to thank our subjects, especially those in the clinical group, for participating in the reported study, as well as three anonymous reviewers for very helpful comments on an earlier version of this manuscript.

\section{References}

Arroyo, Francisco V. 1982. Negatives in context. Journal of Verbal Learning and Verbal Behavior 21: 118-126.

Baltaxe, Christiane A. M. 1977. Pragmatic deficits in the language of autistic adolescents. Journal of Pediatric Psychology 2: 176-180.

Baron-Cohen, Simon. 1988. Social and Pragmatic Deficits in Autism: Cognitive or Affective? Journal of Autism and Developmental Disorders 18: 379-402.

Baron-Cohen, Simon. 1993. From attention-goal psychology to belief-desire psychology: the development of a theory of mind, and its dysfunction. In Simon Baron-Cohen, Helen Tager-Flusberg, and Donald J. Cohen (Hrsg.), Understanding other Minds. Perspectives from Autism. Oxford: Medical Publications. 59-82.

Baron-Cohen, Simon. 2000. Theory of mind and autism: a fifteen year review. In Simon Baron-Cohen, Helen Tager-Flusberg \& Donald J. Cohen (eds.). Understanding other 
minds. Perspectives from developmental cognitive neuroscience. $2^{\mathrm{d}}$ ed. Oxford: Oxford University Press. 3-20.

Baron-Cohen, Simon, Alan M. Leslie, and Uta Frith. 1985. Does the autistic child have a theory of mind? Cognition 21: 37-46.

Baron-Cohen, Simon, Helen Tager-Flusberg, and Donald J. Cohen. 2000. Understanding Other Minds: Perspectives from Autism and Developmental Cognitive Neuroscience. Oxford: Oxford University Press.

Beltran, David, Isabel Orenes, and Carlos Santamaria. 2008. Context effects on the spontaneous production of negation. Intercultural Pragmatics. 5 (4).

Bloom, Lois. 1970. Language Development; Form and Function in Emerging Grammars. Cambridge, MA: MIT Press.

Bonnefon, Jean-Francois, and Gaëlle Villejoubert. 2007. Modus Tollens, Modus Shmollens: Contrapositive reasoning and the pragmatics of negation. Thinking and Reasoning 13: 207-222.

Clark, Herbert H., and Eve V. Clark. 1977. Psychology and Language: An Introduction to Psycholinguistics. San Diego: Harcourt Brace Jovanovich.

Colston, Herbert L. 1999. "Not Good" is "Bad", but "Not Bad" is Not "Good": An Analysis of Three Accounts of Negation Asymmetry. Discourse Processes 28: 237-256.

Dennis, Maureen, Anne L. Lazenby, and Linda Lockyer. 2001. Inferential Language in High-Functioning Children with Autism. Journal of Autism and Developmental Disorders 31: 47-54.

Emerich, David. M., Nancy A. Creaghead, Sandra M. Grether, Dona Murray, and Carol Grasha. 2003. The comprehension of humorous materials by adolescents with highfunctioning autism and Aspergers's syndrome. Journal of Autism and Developmental Disorders 33: 253-257.

Fein, Deborah, Bruce Pennigton, Philip Markowitz, Mark A. Braverman, and Lynn Waterhouse. 1986. Towards a neuropsychological model of infantile autism: Are social deficits primary? Journal of the American Academy of Child Psychiatry 25: 198-212.

Fraenkel, Tamar and Yaacov Schul, Yaacov 2008. The meaning of negated adjectives. Intercultural Pragmatics 5 (4).

Frith, Uta. 1989. Autism: explaining the enigma. Oxford: Blackwell.

Frith, Uta, and Francesca G. E. Happé. 1994. Autism: beyond "theory of mind". Cognition 50: 115-132.

Giora, Rachel. 2006. Anything negatives can do affirmatives can do just as well, except for some metaphors. Journal of Pragmatics 38: 981-1014.

Giora, Rachel, Noga Balaban, Ofer Fein, and Inbar Alkabetz. 2005. Negation as positivity in disguise. In Herbert L. Colston and Albert N. Katz (eds.), Figurative language comprehension: Social and cultural influences. Hillsdale, NJ: Erlbaum. 233-258.

Giora, Rachel, Ofer Fein, Jonathan Ganzi, Ntalie A. Levi, and Hadas Sabah. 2005. On negation as mitigation: The case of negative irony. Discourse Processes 39: 81-100.

Givón, Talmy. 1978. Negation in language: Pragmatics, function ontology. In Peter Cole (ed.), Syntax and semantics Vol. 9: Pragmatics. New York: Academic Press.

Glenberg, Arthur M., David A. Robertson, and Mina C. Johnson-Glenberg. 1999. Not propositions. Journal of Cognitive Systems Research 1: 19-33.

Halliday, Michael, and Zoe L. James. 1993. A quantitative study of polarity and primary tense in the English finite clause. In John M. Sinclair, Michael Hoey, and Gwyneth Fox (eds.), Techniques of Description: Spoken and Written Discourse (A Festschrift for Malcolm Coulthard). London: Routledge. 32-66.

Happé, Francesca G. E. 1993. Communicative competence and theory of mind in autism. A test of relevance theory. Cognition 48: 101-109. 
Happé, Francesca G. E. 1995. Understanding minds and metaphors: Insights from the study of figurative language in autism. Metaphor and Symbolic Activity 10: 275-295.

Happé, Francesca G. E., and Uta Frith. 2006. The Weak Coherence Account: Detailfocused Cognitive Style in Autism Spectrum Disorders. Journal of Autism and Developmental Disorders 36: 5-25.

Heinemann, Wolfgang. 1983. Negation und Negierung. Handlungstheoretische Aspekte einer linguistischen Kategorie. Leipzig: Verlag Enzyklopädie.

Jolliffe, Therese, and Simon Baron-Cohen. 1997. Are people with autism or Asperger's syndrome faster than normal on the Embedded Figures Task? Journal of Child Psychology and Psychiatry 38: 527-534.

Jolliffe, Therese, and Simon Baron-Cohen. 1999a. The strange story test: A replication with high-functioning adults with autism or Asperger syndrome. Journal of Autism and developmental disorders 29: 395-406.

Jolliffe, Therese, and Simon Baron-Cohen. 1999b. A test of central coherence theory: linguistic processing in high-functioning adults with autism or Asperger syndrome: Is local coherence impaired? Cognition 71: 149-185.

Jolliffe, Therese, and Simon Baron-Cohen. 2000. Linguistic processing in high-functioning adults with autism or Asperger's syndrome: Is global coherence impaired? Psychological Medicine 30: 1169-1187.

Kaland, Nils, Annette Møller-Nielsen, Kirsten Callesen, Erik L. Mortensen, Dorte Gottlieb, and Lars Smith. 2002. A new 'advanced' test of theory of mind: evidence from children and adolescents with Asperger syndrome. Journal of Child Psychology and Psychiatry 43: $517-528$.

Kaup, Barbara, Jana Lüdtke, and Rolf A. Zwaan. 2006. Processing negated sentences with contradictory predicates: Is a door that is not open mentally closed? Journal of Pragmatics 38: $1033-1050$.

Kaup, Barbara, Rolf A. Zwaan, and Jana Lüdtke. 2007. The experiential view of language comprehension: How is negation represented? In Franz Schmalhofer and Charles A. Perfetti (eds.), Higher level language processes in the brain: Inference and comprehension processes. Mahwah, NJ: Erlbaum 255-288.

Kelley, Elizabeth, Jennifer J. Paul, Deborah Fein, and L. R. Naigles. 2006. Residual language deficits in optimal outcome children with a history of autism. Journal of Autism and Developmental Disorders 36: 807-828.

Kerbel, Debra, and Pam Grunwel. 1998. A study of idiom comprehension in children with semantic-pragmatic difficulties. PartII: Between groups results and discussion. International Journal of Language and Communication Disorders 33: 23-44.

Lewin, Wiliam H., and Joel Hagaman. 2009. Negated concepts interfere with anaphor resolution. Intercultural Pragmatics 5 (4).

Loukusa, Soile. 2007. The use of context in pragmatics language comprehension in normally developing children and children with Asperger syndrome / high functioning autism. Unpublished dissertation. University of Oulu.

Lüdtke, Jana, and Barbara Kaup. 2006. Context effects when reading negative and affirmative sentences. In Ron Sun (ed.), Proceedings of the 28th annual conference of the cognitive science society. Mahwah, NJ: Lawrence Erlbaum. 1735-1740.

Mehl, Matthias R., and James W. Pennebaker. 2003. The sounds of social life: A psychometric analysis of students' daily social environments and natural conversations. Journal of Personality and Social Psychology 84: 857-870.

Noens, Ilse J. J., and Ina A. van Berckelaer-Onnes. 2005. Captured by details: sense-making, language and communication in autism. Journal of Communication Disorders 38: 123141. 
Norbury, Courtenay F., and Dorothy V. M. Bishop. 2002. Inferential processing and story recall in children with communication problems: a comparison of specific language impairment, pragmatics language impairment and high-functioning autism. International Journal of Language and Communication Disorders 37: 227-251.

Ozonoff, Sally, and Judith N. Miller. 1996. An Exploration of Right-Hemisphere Contributions to the Pragmatic Impairments of Autism. Brain and Language 52: 411-434.

Ramberg, Chris, Stefan Ehlers, Agneta Nydén, Maria Johansson, and Chistopher Gillberg. 1996. Language and pragmatic functions in school-age children on the autism spectrum. European Journal of Disorders of Communication 31: 387-414.

Rumsey, Judith M., and A. P. Hanahan. 1990. Getting it "right": Performance of high functioning autistic adults on a right hemisphere battery. Journal of Clinical and Experimental Neuropsychology 12: 81.

Schmidt, Siegfried J. 1973. Texttheoretische Aspekte der Negation. Zeitschrift für germanistische Linguistik 1: 178-208.

Shah, Amitta, and Uta Frith. 1993. Why Do Autistic Individuals Show Superior Performance on the Block Design Task? Journal of Child Psychology and Psychiatry 34: 13511364.

Shapiro, Theodore, and Richard Kapit. 1978. Linguistic negation in autistic and normal children. Journal of Psycholinguistic Research 7: 337-351.

Sodian, Beate. 2005. Tiefgreifende Entwicklungsstörungen. Autismus. In Peter F. Schlottke, Rainer K. Silbereisen, Silvia Schneider, and Gerhard W. Lauth (eds.). Enzyklopädie der Psychologie. Serie II. Klinische Psychologie. Bd. 5 Störungen im Kindes- und Jugendalter. Göttingen: Hogrefe.

Szatmari, Peter, Lawrence Tuff, M. Allen Finlayson, and Giampierro Bartolucci. 1990. Asperger's syndrome and autism: Neurocognitive aspects. Journal of the American Academy of Child and Adolescent Psychiatry 29: 130-136.

Tager-Flusberg, Helen. 1981. On the Nature of Linguistic Functioning in Early Infantile Autism. Journal of Autism and Developmental Disorders 11: 45-56.

Tager-Flusberg, Helen. 1985. Psycholinguistic Approaches to language and communication in autism. In Eric Schopler and Gary B. Mesibov (eds.). Current Issues in autism. New York: Kluwer. 69-88.

Tager-Flusberg, Helen. 1996. Brief report: Current theory and research on language and communication in autism. Journal of Autism and Developmental Disorders 26: 169-172.

Tager-Flusberg, Helen. 1999. A psychological approach to understanding the social and language impairments in autism. International Review of Psychiatry 11: 325-334.

Van Selst, Mark, and Pierre Jolicoeur. 1994. A Solution to the effect of sample size on outlier elimination. The Quarterly Journal of Experimental Psychology 47A: 631-650.

Wason, Peter C. 1965. The contexts of plausible denial. Journal of Verbal learning and Verbal Behavior 4: 7-11.

Wason, Peter C. 1972. In real life negatives are false. Logique et Analyse 15: 17-38.

Watson, Judith M. 1979. Referential description by children in negative form. British Journal of Psychology 70: 199-204.

Wimmer, Heinz, and Josef Perner. 1983. Belief about beliefs: representation and constraining function of wrong beliefs in young children's understanding of deception. Cognition 13: 103-128. 